\title{
Inverse modelling for mercury over Europe
}

\author{
Y. Roustan and M. Bocquet \\ Centre d'Enseignement et de Recherche en Environnement Atmosphérique, Joint laboratory École Nationale des Ponts et \\ Chaussées/EDF R\&D, avenue Blaise Pascal, 77455 Champs sur Marne, France
}

Received: 12 October 2005 - Published in Atmos. Chem. Phys. Discuss.: 30 January 2006

Revised: 3 May 2006 - Accepted: 15 May 2006 - Published: 24 July 2006

\begin{abstract}
The fate and transport of mercury over Europe is studied using a regional Eulerian transport model. Because gaseous elemental mercury is a long-lived species in the atmosphere, boundary conditions must be properly taken into account. Ground measurements of gaseous mercury are very sensitive to the uncertainties attached to those forcing conditions. Inverse modelling can help to constrain the forcing fields and help to improve the predicted mercury concentrations. More generally, it allows to reduce the weaknesses of a regional model against a global or hemispherical model for such diffuse trace constituent. Adjoint techniques are employed to relate rigorously and explicitly the measurements to the forcing fields. This way, the inverse problem is clearly defined. Using EMEP measurements of gaseous mercury and performing the inversions, it is shown that boundary conditions can be improved significantly as well as the forecast concentrations. Using inverse modelling to improve the emission inventory is however much more difficult. Indeed, there are currently not enough mercury monitoring stations, and they are located far away from the center of Europe.
\end{abstract}

\section{Introduction}

Gaseous elemental mercury (GEM) makes up more than 95\% of the mass of atmospheric mercury (Ryaboshapko et al., 2002), but mercury can also be found under oxidised forms, both in gaseous and aqueous phases and possibly linked to the particulate matter. Life times of mercury species strongly vary from one year for GEM (Lindqvist and Rodhe, 1985), days to weeks for mercury adsorbed/absorbed to particulate matter, and hours to days for oxidised gaseous species (Seigneur et al., 2003) operationally defined as reactive gaseous mercury (RGM). These life times are obviously

Correspondence to: Y. Roustan

(roustan@cerea.enpc.fr) driven by the rates of dry and wet deposition which are in turn governed by physical and chemical properties of the species.

Owing to its long life time, mercury is considered as a global pollutant. Hence the Chemistry Transport Models (CTM) currently used to simulate atmospheric mercury fate and transport run on a global domain (Seigneur et al., 2001) or a hemispherical one (Ilyin et al., 2002; Christensen et al., 2004). Such models proved well suited to the study of transboundary pollution. Nevertheless regional models remain suitable for impact studies needing finer spatial resolution whereas global model may have too coarse resolution to get accurate estimations of local deposition fluxes. Some simulations are still performed within a restricted domain (Lin and Tao, 2003; Bullock and Brehme, 2002), and generally stand as a first step in atmospheric mercury model development.

Consequently to its long life time GEM is rather homogeneously mixed in the atmosphere. Typical concentrations are in the range of one to two $\mathrm{ng} \mathrm{m}^{-3}$. With modelling issues in mind, this behaviour suggests that boundary conditions for a limited area model are crucial. As a consequence a regional model can account for mercury dispersion only if boundary conditions are properly addressed. This may be achieved though inverse modelling. Because of the linearity of dispersion and all physical processes of mercury (dry deposition, wet scavenging, chemistry), the forecasted concentrations can be related explicitly to the forcing fields (in particular boundary conditions). In the case of atmospheric mercury, this has been recently carried out using adjoint techniques (Roustan and Bocquet, 2006).

In Sect. 2 of this paper, the mercury dispersion model used is detailed. A few results about the global budget of mercury in a regional domain is given, in order to emphasise the role of mercury exchanges in and out of the domain. In Sect. 3, the way adjoint methods should be used to establish the inverse problem is advocated, both for the continuous and the numerical (discrete) models. In Sect. 4, an inverse modelling approach building on the tools introduced previously

Published by Copernicus GmbH on behalf of the European Geosciences Union. 


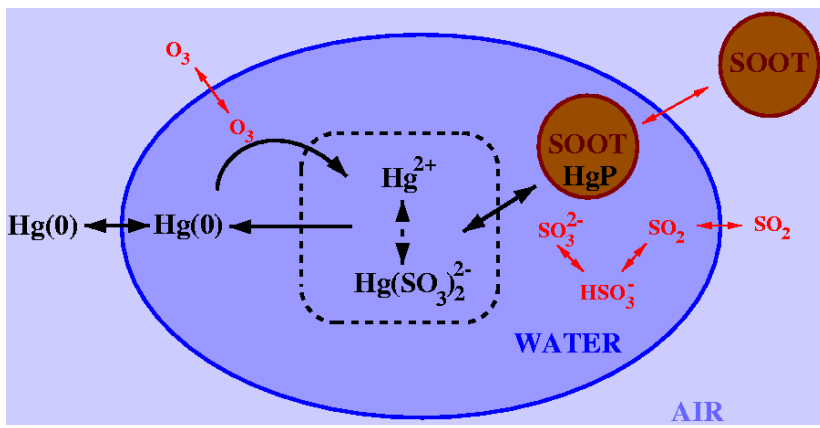

Fig. 1. Schematic of the Petersen et al. (1995) mercury chemistry model.

and which aims mainly at improving boundary conditions is tested. In Sect. 5, the inverse modelling methodology is generalised and tested with a complex chemistry scheme, accounting for oxidised mercury species. Conclusions are given in Sect. 6.

\section{Simulating mercury over Europe}

The following equation describes the transport and fate of mercury concentration, $c$, under the influence of well identified atmospheric processes:

$$
\frac{\partial c}{\partial t}+\operatorname{div}(\boldsymbol{u} c)-\operatorname{div}(\mathbf{K} \nabla c)+\Lambda c=\sigma
$$

The temporal evolution of mercury concentration is governed by, from left to right in Eq. (1), the advection by the wind field $\boldsymbol{u}$, the turbulent diffusion (characterised by the eddy diffusion tensor $\mathbf{K}$ ), the wet scavenging including a parameterisation of the chemistry (space and time varying coefficient $\Lambda)$ and finally sources $(\sigma)$.

Dry deposition (with $v_{d}$ the dry deposition velocity) and surface emission $(E)$ are enforced as a ground boundary condition (the normal surface vector, $\boldsymbol{n}$, is outward oriented):

$(\mathbf{K} \nabla c) \cdot \boldsymbol{n}=E-v_{d} c$.

\subsection{Physical and chemical parameterisations}

The chemistry model which will be used has been proposed in Petersen et al. (1995). In this model elemental mercury is considered as a passive tracer in gaseous phase but as a reactive chemical in the aqueous phase. Ozone is the only oxidant species accounting for the oxidised mercury formation. Oxidised mercury in aqueous phase can form a complex with sulfite ions or it can be adsorbed by particulate matter. The complex may either be decomposed and give elemental mercury or it may be adsorbed by particulate matter in turn (see Fig. 1).

More reactions and species are represented in currently developed models (Ryaboshapko et al., 2002). The aim is to get better evaluation of oxidised species concentration in order to improve deposition flux patterns. Yet, this work will mostly require a correct modelling of the GEM concentration field. Forced concentration fields are used for ozone and soot particles. As mentioned previously, this model is based on several equilibria hypotheses, which allows to represent chemistry through a scavenging ratio.

An interesting point is that the chemistry-scavenging term in Eq. (1) is linear (so are the advection and diffusion terms). In practice, numerically modelled GEM nearly behaves like a passive tracer.

Wet scavenging represent pollutant mass transfer from the atmosphere to the soil during precipitation events. The mass could be collected by cloud drops (in cloud scavenging) or rain drops (below-cloud scavenging).

GEM is also removed from the atmosphere by dry deposition. Often dry deposition is decomposed into three consecutive processes that bring pollutant from atmosphere to soil surface under dry conditions (Wesely and Hicks, 2000). The first one is the turbulent diffusion that is the dominant process in most of the layer between the height where dry deposition velocity is estimated and the soil. In the quasi-laminar layer gaseous molecular diffusion becomes the major process. The mass transfer processes from the air to the canopy completes the removal mechanism.

A common big-leaf deposition resistance model is used to compute dry deposition velocities. Resistances parameterisation are inspired by Baer and Nester (1992) with some improvements, in particular for the quasi-laminar boundary resistance over sea (Hicks and Liss, 1976) and the canopy water content consideration in canopy resistance (Brook et al., 1999). Those parameterisations are further detailed in Roustan et al. (2005).

\subsection{A regional domain model}

The transport and physics of mercury is meant here to be simulated over Europe. The domain which is considered (Fig. 2) extends in space from $12.375^{\circ} \mathrm{W}$ to $37.125^{\circ} \mathrm{E}$ in longitude and from $36^{\circ} \mathrm{N}$ to $72^{\circ} \mathrm{N}$ in latitude (Europe). Direct and backward (adjoint modelling) simulations are performed for the year 2001. A constant space step of $1.125^{\circ}$ is taken along longitude and latitude for the horizontal grid of $44 \times 32$ cells, respectively. The 14 vertical levels cover atmosphere from the ground to $5233 \mathrm{~m}$ in relative height.

The domain is designated by $\Omega$ and it is the product of its spatial and temporal components $\Omega=\mathcal{D} \times[0, \tau]$. The boundaries of the domain $\Omega$ are denoted $\partial \Omega_{0}, \partial \Omega_{\tau}, \partial \Omega_{b}, \partial \Omega_{t}$, $\partial \Omega_{n}, \partial \Omega_{s}, \partial \Omega_{w}$ and $\partial \Omega_{e}$, for respectively the initial, final, surface, top, North, South, West and East boundaries. The boundary of the space domain is denoted $\partial \mathcal{D}$. A distinction is to be made between border interfaces where the wind is incoming, and border interfaces where it is outgoing. Hence the spatial boundary splits into $\partial \mathcal{D}=\partial \mathcal{D}_{+} \cup \partial \mathcal{D}_{-}$ ( + means incoming, and - means outgoing). Note that 
Table 1. Elemental mercury mass budget over Europe for year 2001, using the Petersen's chemistry. Mass figures are truncated to their first decimal. The last line lays the final mass budget equation.

\begin{tabular}{|c|c|c|c|c|}
\hline \multicolumn{2}{|c|}{ masses (in tons) } & incoming & outgoing & sum \\
\hline \multicolumn{4}{|c|}{ initial mass $\left(\mathrm{M}_{i}\right) \partial \Omega_{0}$} & 107 \\
\hline \multicolumn{5}{|c|}{ final mass $\left(\mathbf{M}_{f}\right) \partial \Omega_{\tau}$} \\
\hline west flux & $\partial \Omega_{w}$ & 7713 & 1752 & 5961 \\
\hline east flux & $\partial \Omega_{e}$ & 1222 & 6353 & -5131 \\
\hline south flux & $\partial \Omega_{s}$ & 2411 & 3794 & -1383 \\
\hline north flux & $\partial \Omega_{n}$ & 1324 & 1618 & -294 \\
\hline top flux & $\partial \Omega_{t}$ & 6328 & 5722 & 606 \\
\hline \multicolumn{5}{|c|}{ surface emission $\partial \Omega_{b}$} \\
\hline anthropogenic & & 73 & & 73 \\
\hline natural & & 100 & & 100 \\
\hline reemission & & 34 & & 34 \\
\hline \multicolumn{5}{|c|}{ volume emission $\Omega$} \\
\hline $\begin{array}{l}\text { anthropogenic } \\
\text { natural } \\
\text { reemission }\end{array}$ & & 73 & & 73 \\
\hline dry deposition & \multicolumn{2}{|l|}{$\partial \Omega_{b}$} & 28 & -28 \\
\hline wet deposition & \multicolumn{2}{|l|}{$\partial \Omega_{b}$} & negligible & negligible \\
\hline \multicolumn{4}{|c|}{$\mathrm{M}_{f}-\mathrm{M}_{i}-\Sigma$ flux } & 0.1 \\
\hline
\end{tabular}

this decomposition is time-dependent. We will also note $\partial \Omega_{ \pm}=\bigcup_{t} \partial \mathcal{D}_{ \pm}[t]$. Finally, $\partial \mathcal{D}$, the spatial boundary of $\mathcal{D}$, is made up of the bottom (surface), top, North, South, West and East borders, $\partial \mathcal{D}_{b}, \partial \mathcal{D}_{t}, \partial \mathcal{D}_{n}, \partial \mathcal{D}_{s}, \mathcal{D}_{w}$, and $\partial \mathcal{D}_{e}$, respectively.

It is a rather limited horizontal area in comparison to what is currently done to study mercury impact over Europe (Ilyin et al., 2003). Because of its long atmospheric life time GEM is considered to be a global pollutant, hence should be studied by means of a global model. Since the magnitude order of GEM residence time and inter-hemispheric exchange time are quite similar hemispheric model may be relevant. However such models need much more data and computing time to perform simulation with relatively coarse spatial resolution. One aim of this work is to evaluate the feasibility and the interest of inverse modelling on boundary conditions to avoid use of global and hemispheric models.

\subsection{Mercury mass budget over Europe}

A mass budget is a diagnosis tool to test the accuracy of the numerical transport model. It helps ensuring that numerics are under control. In addition it provides with data on the magnitude of transboundary mercury fluxes, as well as ground emissions and sources. It is a first albeit gross view on the potential drawbacks of a limited area model versus a global or hemispherical model.

A simulation has been performed for the year 2001, using the CTM POLAIR3D, whose characteristics will further

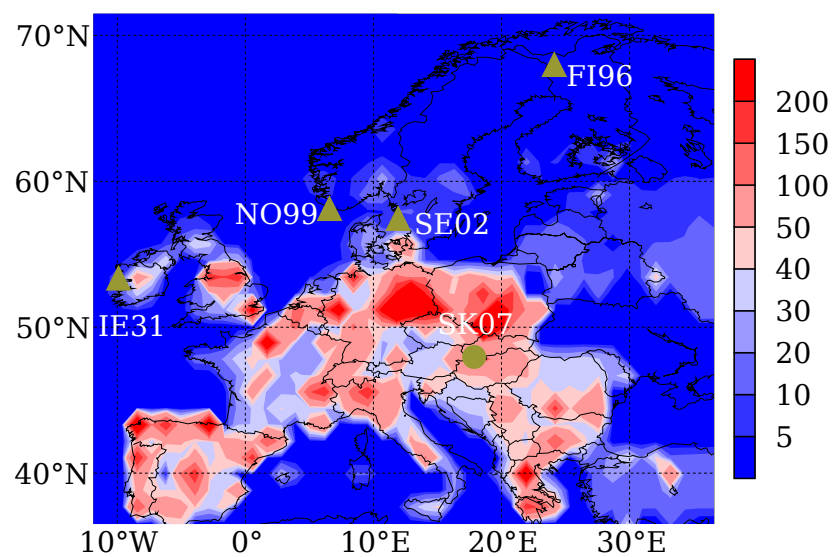

Fig. 2. Mean annual emissions (in $\mu \mathrm{g} \mathrm{m}^{-2} \mathrm{yr}^{-1}$ ) over the domain $\mathcal{D}$. Symbols $\boldsymbol{\Delta}$ and $\bullet$ indicate EMEP gaseous mercury monitoring stations and Topolniky station, respectively.

be detailed in Sect. 3.2. The emission data (see Fig. 2) are those provided by the Meteorological Synthesising Centre East (MSC-E) for the year 2001, which is one of the European Monitoring and Evaluation Program (EMEP) centre (http://www.msceast.org/). Mercury emissions are usually classified into three types, anthropogenic, natural and reemission (Ryaboshapko et al., 1998). For the simulation anthropogenic emissions are split into ground emission and sources (emissions in the bulk) at the second vertical level. Meteorological fields are derived from re-analysis of the European Centre for Medium-Range Weather Forecasts (ECMWF) with a six hours frequency. Vertical wind fields are diagnosed in order to ensure mass conservation under the incompressible atmosphere hypothesis. A homogeneous initial concentration of $1.5 \mathrm{ng} \mathrm{m}^{-3}$ is taken in the bulk. The simulation time step is $900 \mathrm{~s}$, data are linearly interpolated between each data time step. The following uniform boundary conditions are implemented: $1.75 \mathrm{ng} \mathrm{m}^{-3}$ at West, $1.7 \mathrm{ng} \mathrm{m}^{-3}$ at East, $1.5 \mathrm{ng} \mathrm{m}^{-3}$ at South and $1.42 \mathrm{ng} \mathrm{m}^{-3}$ at North. These values are proposed by the MSC-E in a first approach. In addition a value of $1.6 \mathrm{ng} \mathrm{m}^{-3}$ at the top of the domain was chosen. For each boundary of the domain total mass fluxes have been computed. For each type of emission, the total released mass is given. The initial and final mercury masses present in the domain are also part of the budget. Those fluxes are listed in Table 1.

At first this budget confirms the consequent contribution of boundary fluxes to the GEM concentration in the bulk, especially on the western border, which is consistent with the average atmospheric circulation over Europe from West to North-East. Secondly the initial and final masses are similar and relatively low in comparison to advected fluxes.

In this respect, Fig. 3 unveils that the initial conditions are almost forgotten after a two weeks spin-up time. Therefore the final mass is probably mainly ascribable to meteorological, boundary conditions and dynamical input of mercury. 
The absolute values of fluxes seem far superior to the mass injected by emissions. Having in mind to extract information from such a regional model about (for instance) the impact of emissions in Europe, this last remark may sound compromising. However those figures do not give direct information about the sensitivity of mercury concentrations near the ground. Contributions of surface emissions and sources appear moderate. However since the spatial origin of these fluxes is close to the ground one can expect to find a relatively high sensitivity to surface concentrations. The fluxes at the top of the domain are also important. However the exchange surface is much more extended than those of the other domain boundaries. Since mass exchanges between the top, belonging to the free troposphere, and the surface, in the atmospheric boundary layer, are rather limited, it seems rational to assume there is little consequence on surface concentrations. The weakness of the chemistry mechanism which largely underestimates the wet deposition flux is also shown, but in a first approach this is not a worrying point for now.

The budget is theoretically balanced. This has been checked numerically, with a very moderate unbalance of $0.1 \mathrm{t}$ of elemental mercury.

\section{Adjoint transport in an open domain}

As for any inverse problems, we need to establish the link between the output (the measurements) and the forcing fields. This can be carried out rigorously with adjoint analytical and numerical techniques.

\subsection{Continuous analysis}

Full details of the calculation can be found in Roustan and Bocquet (2006), as well as references to the use of adjoint techniques in air quality models. Here we merely give the definitions and results.

A concentration measurement (of value $\mu_{i}$, performed on site $i$ ) is characterised by a sampling function $\pi_{i}: \Omega \rightarrow \mathbb{R}$, such that $\int_{\Omega} \mathrm{d} t \mathrm{~d} \boldsymbol{x} \pi_{i}(\boldsymbol{x}, t)=1$ and

$\mu_{i}=\int_{\Omega} \mathrm{d} t \mathrm{~d} \boldsymbol{x} \pi_{i}(\boldsymbol{x}, t) c(\boldsymbol{x}, t)$.

Let $c_{i}^{*}$ be a solution of the retro-transport equation, forced by $\pi_{i}$ :

$-\frac{\partial c_{i}^{*}}{\partial t}-\operatorname{div}\left(\boldsymbol{u} c_{i}^{*}\right)-\operatorname{div}\left(\mathbf{K} \nabla c_{i}^{*}\right)+\Lambda c_{i}^{*}=\pi_{i}$.

The justification for introducing $c_{i}^{*}$ will appear thereafter. To characterise $c_{i}^{*}$ completely, boundary and initial conditions must be specified.

As is clearly seen from Eq. (4), the adjoint solution $c_{i}^{*}$ corresponds to a transport backward in time. The wind field is the opposite of the direct model wind field. As a consequence, an outgoing (from the domain $\mathcal{D}$ ) wind flow for the direct model is actually an incoming wind flow for the adjoint model. In order to specify the advective incoming mercury, we therefore need to specify its concentration on $\partial \mathcal{D}_{-}$at any time. For simplicity,

$\forall(\boldsymbol{x}, t) \in \partial \Omega_{-}, \quad c_{i}^{*}(\boldsymbol{x}, t)=0$,

is assumed (among other possible consistent choices).

In addition, the diffusive fluxes $-\mathbf{K} \nabla c$ and $-\mathbf{K} \nabla c_{i}^{*}$ at the boundary $\partial \mathcal{D}$ are supposed both negligible when compared to the advected flux, or imposed (when possible) as 0 . However at the surface, $-\mathbf{K} \nabla c$ is no different than the surface emission $\boldsymbol{J}$. In a similar fashion, $-\mathbf{K} \nabla c_{i}^{*}$ could be chosen at the surface, a given value $\boldsymbol{J}_{i}^{*}$ which is to be prescribed later on.

Finally, the adjoint concentration field $c_{i}^{*}$ is set to be null at initial time, which is $t=\tau$ (simplest choice over many possible).

Using this completely specified adjoint solution it can be shown that (Roustan and Bocquet, 2006)

$$
\begin{aligned}
\mu_{i}= & \int_{\Omega} \mathrm{d} t \mathrm{~d} \boldsymbol{x} c_{i}^{*} \sigma+\int_{\partial \Omega_{0}} \mathrm{~d} \boldsymbol{x} c_{i}^{*} c \\
& +\int_{\partial \Omega_{b}} \mathrm{~d} t \mathrm{~d} \boldsymbol{S} \cdot\left(c \boldsymbol{J}_{i}^{*}-c_{i}^{*} \boldsymbol{J}\right)-\int_{\partial \Omega_{+}} \mathrm{d} t \mathrm{~d} \boldsymbol{S} \cdot\left(c_{i}^{*} c \boldsymbol{u}\right) .
\end{aligned}
$$

Let us denote $\boldsymbol{n}$ the unit vector orthogonal to the boundary, oriented outward $(\mathrm{d} \boldsymbol{S}=\mathrm{d} S \boldsymbol{n})$. In POLAIR3D, $\boldsymbol{J} \cdot \boldsymbol{n}$ stands actually for $v^{\text {dep }} c_{\mid b}-E$. That is why the choice $\boldsymbol{J}_{i}^{*} \cdot \boldsymbol{n}=v^{\text {dep }} c_{i \mid b}^{*}$ allows for a simplification in the kernel:

$\int_{\partial \Omega_{b}} \mathrm{~d} t \mathrm{~d} \boldsymbol{S} \cdot\left(c \boldsymbol{J}_{i}^{*}-c_{i}^{*} \boldsymbol{J}\right) \rightarrow-\int_{\partial \Omega_{b}} \mathrm{~d} t \mathrm{~d} \boldsymbol{S} \cdot\left(c_{i}^{*} \boldsymbol{E}\right)$,

with $\boldsymbol{E}=-\boldsymbol{E} \boldsymbol{n}$. Therefore this specific choice of the adjoint solution makes the connection between the output and the surface emission clearer. In particular, this choice of adjoint solution stipulates dry deposition is to be taken into account in the calculation of the retroplume.

Equations (6) and (7) make clear links between,

- the surface emission $\boldsymbol{E}$,

- the volume emission $\sigma$,

- the initial concentrations $c$ on $\partial \Omega_{0}$,

- the boundary concentrations $c$ on $\partial \Omega_{+}$,

and the output, the modelised observation $\mu_{i}$. This decomposition explains a posteriori why the abstract function $c_{i}^{*}$ was introduced.

\subsection{Application to a numerical transport model}

To perform numerical investigation, the domain $\Omega$ is discretised into a grid (seen as a set of cells) $\bar{\Omega}=\bigcup_{k} \Omega_{k}$, where $\Omega_{k}$ is a grid-cell. $k$ indexes the mesh, with $k=1, \cdots, N_{x} N_{y} N_{z} N_{t}$. A border cell belongs to one of the 
grid boundaries $\overline{\partial \Omega}_{0}, \overline{\partial \Omega}_{\tau}, \overline{\partial \Omega}_{b}, \overline{\partial \Omega}_{t}, \overline{\partial \Omega}_{n}, \overline{\partial \Omega}_{s}, \overline{\partial \Omega}_{w}$, and $\bar{\partial}_{e}$. Boundaries $\overline{\partial \Omega}_{ \pm}$are the grid-cells forming the onelayer boundaries of $\bar{\Omega}$.

In this paper, we apply our methods using the Chemistry Transport Model POLAIR3D (see Sportisse et al., 2002; Sartelet et al., 2002; Boutahar et al., 2004). The two chemistry modules implemented here are presented in Roustan et al. (2005). The numerical code is based on a first order time splitting algorithm allowing to separate temporally chemistry (when relevant), advection and diffusion. The advection scheme is a third-order Direct Space Time (DST) scheme (Spee, 1998) with the Koren-Sweby flux limiter function. Its related temporal scheme is explicit. The diffusion scheme is a spatially centred three point scheme (for each direction). Its related temporal scheme is a semi-implicit Rosenbrock scheme.

The adjoint analysis can be carried out onto the numerical model. A scheme without approximation would require to compute the adjoint of the numerical model to obtain the adjoint numerical solutions. It is however easier to discretise the adjoint transport equation, which should be considered as a reasonable approximation in this context (Roustan and Bocquet, 2006). Detailed calculations show that the adjoint of POLAIR3D would be POLAIR3D itself, antisymmetric fields such as wind fields being reversed, if not for occasional non-linearity and if not the $K_{z}$ time-dependence (M. Bocquet, unpublished). The error entailed by this approximation has been estimated. The result will given in the more intricate case of a complex chemistry model (Sect. 5).

The results of the adjoint analysis sum up to the formula:

$$
\begin{aligned}
\mu_{i}= & \sum_{k \in \bar{\Omega}} c_{i, k}^{*} \sigma_{k}+\sum_{k \in \bar{\partial}_{0}} c_{i, k}^{*} c_{k} \\
& +\sum_{k \in \bar{\partial}_{b}}\left(c_{i, k}^{*} J_{k}-c_{k} J_{i, k}^{*}\right)+\sum_{k \in \bar{\partial}_{+}} c_{i, k}^{*} F_{k},
\end{aligned}
$$

very similar to its continuous counterpart. Space and time volume elements which appear in the discretised sums have been integrated into the sources $\sigma_{k}$, space volume elements have been integrated into the initial concentrations $c_{k \mid 0}$, whereas surface elements have been integrated into the emissions $J_{i, k}^{*}=-\boldsymbol{n} \cdot \boldsymbol{F}_{i, k}^{*}$ and $\boldsymbol{J}_{k}=-\boldsymbol{n} \cdot \boldsymbol{J}_{k}$, and the advected fluxes $F_{k}=-\boldsymbol{n} \cdot \boldsymbol{F}_{k}$. Therefore, they all are expressed in units of mass. The numerical advected flux $F_{k}$ could be specified precisely in terms of boundary concentrations and wind fields, with the details of the adjoint calculations. It is positive by definition on $\partial \Omega_{+}$.

In the case where $\boldsymbol{J}_{i}^{*} \cdot \boldsymbol{n}=v^{\text {dep }} c_{i \mid b}^{*}$, Eq. (8) simplifies to

$$
\begin{aligned}
\mu_{i}= & \sum_{k \in \bar{\Omega}} c_{i, k}^{*} \sigma_{k}+\sum_{k \in \bar{\partial}_{0}} c_{i, k}^{*} c_{k} \\
& +\sum_{k \in \bar{\partial}_{b}} c_{i, k}^{*} E_{k}+\sum_{k \in \bar{\partial}_{+}} c_{i, k}^{*} F_{k} .
\end{aligned}
$$

Table 2. Contributions to the EMEP monitoring stations measurements of the West, East, North and South incoming mercury, and the emissions of all kinds, in $\mathrm{ng} \mathrm{m}^{-3}$, as simulated with the simple scheme model.

\begin{tabular}{ccccccc}
\hline Station & West & East & North & South & Emiss. & Total \\
\hline $\begin{array}{c}\text { Mace Head } \\
\text { (IE31) }\end{array}$ & 1.566 & 0.049 & 0.105 & 0.008 & 0.163 & 1.900 \\
$\begin{array}{c}\text { Pallas } \\
\text { (FI96) }\end{array}$ & 0.472 & 0.514 & 0.590 & 0.005 & 0.061 & 1.697 \\
$\quad \begin{array}{l}\text { Lista } \\
\text { (NO99) }\end{array}$ & 1.214 & 0.148 & 0.285 & 0.022 & 0.228 & 1.950 \\
$\begin{array}{l}\text { Rörvik } \\
\text { (SE02) }\end{array}$ & 1.166 & 0.203 & 0.272 & 0.024 & 0.342 & 2.066 \\
\hline
\end{tabular}

This equation clearly establishes the connexion between modelised observation $\mu_{i}$, and the forcing fields. We will use it extensively in the following.

\section{Towards inverse modelling of mercury}

The adjoint techniques which have been introduced in Sect. 3 are necessary technical tools for inverse modelling studies in a systematic approach. They allow to establish the cornerstone relations between data and forcing conditions to be inverted: Eq. (9).

Inverse modelling of mercury can serve two purposes. The first one is the inversion of sources or emissions in order to improve inventories of emissions and sinks and more generally the budget of mercury. However, this might be beyond the scope of this paper, as will be seen. The second one consists in improving boundary conditions enforced and which, as observed, is crucial for the quality of the modelling in a regional domain. This is the main purpose of this work.

\subsection{Improving annual mean boundary conditions}

Let us see how to proceed on an example of interest.

\subsubsection{The boundary conditions problem}

The monthly averaged (therefore possibly annual) measurements of elemental mercury for the four following Nordic EMEP stations are available: Mace Head (IE31 in the EMEP nomenclature), Pallas (FI96), Lista (NO99), and Rörvik (SE02). As suggested, and referring to our air limited domain, those stations are very much influenced by the West, East, or North incoming fluxes, and little by European sources. This can be checked on Table 2 (the incoming flux from the top is negligible and not reported in this table). In the following, the South and "top" fluxes will not be chosen as variables to invert because the sensitivity of the modeled measurements to these forcing fields are to weak. Therefore 


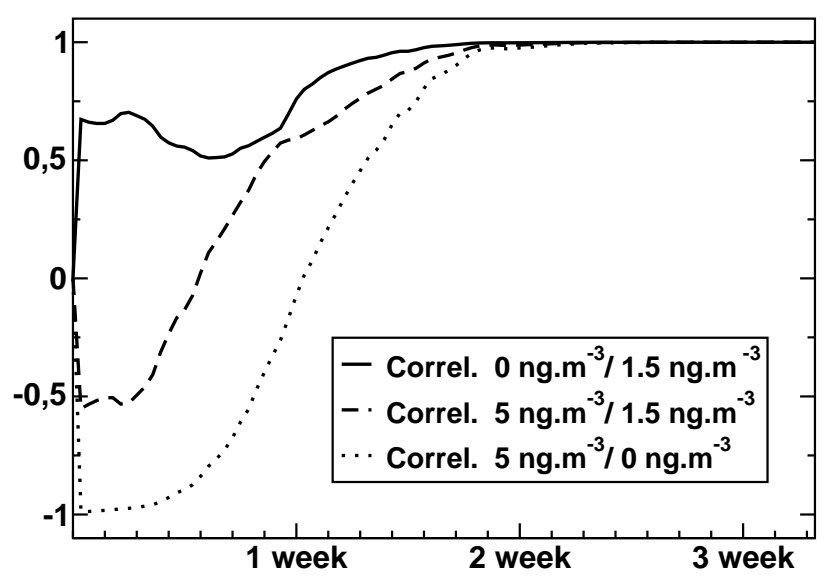

Fig. 3. Influence of initial conditions: spatial correlation between simulation results with different initial bulk conditions, $0 \mathrm{ng} \mathrm{m}^{-3}$, $1.5 \mathrm{ng} \mathrm{m}^{-3}$ (the reference case) and $5 \mathrm{ng} \mathrm{m}^{-3}$.

those parameters cannot be reliably inverted with the observations at our disposal presently.

We could assume uniform boundary conditions on the West, East and North faces of the domain as was done so far. Alternatively one can use non-uniform climatologies for the boundary conditions. They are built by linear interpolation from annual mean concentration fields resulting from simulations performed by the EMEP MSC-E team for year 2001 and then 2002. In this way a more realistic spatial variability is introduced, but the boundary conditions are kept constant in time.

The related fields will be called $c^{\mathrm{W}}, \boldsymbol{c}^{\mathrm{N}}$, and $c^{\mathrm{E}}$. On Fig. 4 are given the monthly averaged concentrations of elemental mercury for the four sites obtained through observation, as well as direct simulations using EMEP uniform boundary conditions for year 2001, EMEP climatic boundary conditions for year 2001, and EMEP climatic boundary conditions for year 2002. Meteorological and emissions data for year 2001 are used to perform these simulations. It is then obvious on the graphs that it is much better to use the 2002 EMEP boundary conditions because of a better overall bias.

\subsubsection{The need for a background term}

Because the inverse problem related to boundary conditions is ill-conditioned, it is important to use a background term which would penalise any too strong departure from the background. A typical background information would be given by first-guess climatologies, denoted $\boldsymbol{b}^{\mathrm{W}}, \boldsymbol{b}^{\mathrm{N}}$, and $\boldsymbol{b}^{\mathrm{E}}$, and a background covariance matrix denoted $\mathbf{B}$, describing a priori their typical fluctuations. Estimating the observation error covariance matrix $\mathbf{R}$ is realistic. However, estimating $\mathbf{B}$ is much more problematic. It is therefore wise to introduce a scalar parameter $\gamma$ such that the background covariance matrix is actually $\gamma^{-1} \mathbf{B}$. It will be estimated later on through a simple cross-validation approach.

\subsection{Inverting annual means boundary conditions}

One improvement would be to allow for three degrees of freedom, so that the boundary conditions could be $\lambda_{\mathrm{W}} c^{\mathrm{W}}$, $\lambda_{\mathrm{N}} c^{\mathrm{N}}$, and $\lambda_{\mathrm{E}} c^{\mathrm{E}}$, with $\lambda_{\mathrm{W}}, \lambda_{\mathrm{N}}, \lambda_{\mathrm{E}}$, three scaling parameters to be determined.

The sensitivity of one of the measurement $\mu_{i}$ to the scalar $\lambda_{\mathrm{f}}$ is

$\frac{\delta \mu_{i}}{\delta \lambda_{\mathrm{f}}}=\sum_{k \in \bar{\partial}_{+} \cap \bar{\partial}_{\mathrm{f}}} c_{i, k}^{*} c_{k}^{\mathrm{f}} u_{k}$,

where f stands for $\mathrm{W}, \mathrm{N}$ or $\mathrm{E}$.

The set of measurements $\mu_{i}$ to be assimilated is the monthly averaged concentrations on the site Mace Head and on the site Pallas. Those correspond to $p=24$ measurements. Given these measurements, one would like to assess the values of the three boundary conditions. Define the $24 \times 3$ matrix

$[\mathbf{H}]_{i, \mathrm{f}}=\frac{\delta \mu_{i}}{\delta \lambda_{\mathrm{f}}}$,

where $\mathrm{f}$ is $\mathrm{W}, \mathrm{N}$ or $\mathrm{E}$. Let $\boldsymbol{\mu}$ be the vector of the twentyfour observations, and let $\boldsymbol{h}$ be the vector whose component $i$ is the (presumably known) contributions from all origin except incoming fluxes from West, East and North. Boundary conditions are stored in the vector $\lambda: \lambda=\left(\lambda_{\mathrm{W}}, \lambda_{\mathrm{N}}, \lambda_{\mathrm{E}}\right)^{T}$. Estimating them would imply minimising the discrepancy from the predicted concentrations to the observed ones. As mentioned earlier, it could also incorporate a background information, which tells one's confidence in the climatological boundary conditions, on a priori grounds. A solution to this problem would therefore be the minimum of the cost function

$$
\begin{aligned}
J= & \frac{1}{2}[\boldsymbol{\mu}-\boldsymbol{h}-\mathbf{H} \boldsymbol{\lambda}]^{T} \mathbf{R}^{-1}[\boldsymbol{\mu}-\boldsymbol{h}-\mathbf{H} \boldsymbol{\lambda}] \\
& +\frac{\gamma}{2}\left[\lambda-\lambda_{b}\right]^{T} \mathbf{B}^{-1}\left[\boldsymbol{\lambda}-\boldsymbol{\lambda}_{b}\right],
\end{aligned}
$$

where the first term of the right-hand side represents departure from the observations. The second term represents departure from the background. $\mathbf{R}$ is the observation error covariance matrix. The observation error is generally considered to be less than $10 \%$ (Ryaboshapko et al., 2003). Therefore the value $0.1 \mathrm{ng} \mathrm{m}^{-3}$ is chosen to represent this. The diagonal matrix $\mathbf{R}$ is then defined by $[\mathbf{R}]_{i j}=\delta_{i j} 0.01$ (in $\left.\mathrm{ng}^{2} \mathrm{~m}^{-6}\right)$.

B is the background covariance matrix. $\lambda$ is likely to be a three-vector of components 1 , if one trusts the climatology. The genuine physical first guesses are $\lambda_{b}^{\mathrm{W}} \boldsymbol{b}^{\mathrm{W}}, \lambda_{b}^{\mathrm{N}} \boldsymbol{b}^{\mathrm{N}}$, and $\lambda_{b}^{\mathrm{E}} \boldsymbol{b}^{\mathrm{E}}$, but background information can be "included" in B. We assume firstly that the error on the background term is not correlated from domain boundary to domain boundary. Secondly this error is assumed of the same order as the observation one $\left(0.1 \mathrm{ng} \mathrm{m}^{-3}\right)$. We define the diagonal terms of the matrix $\mathbf{B}$ by: 

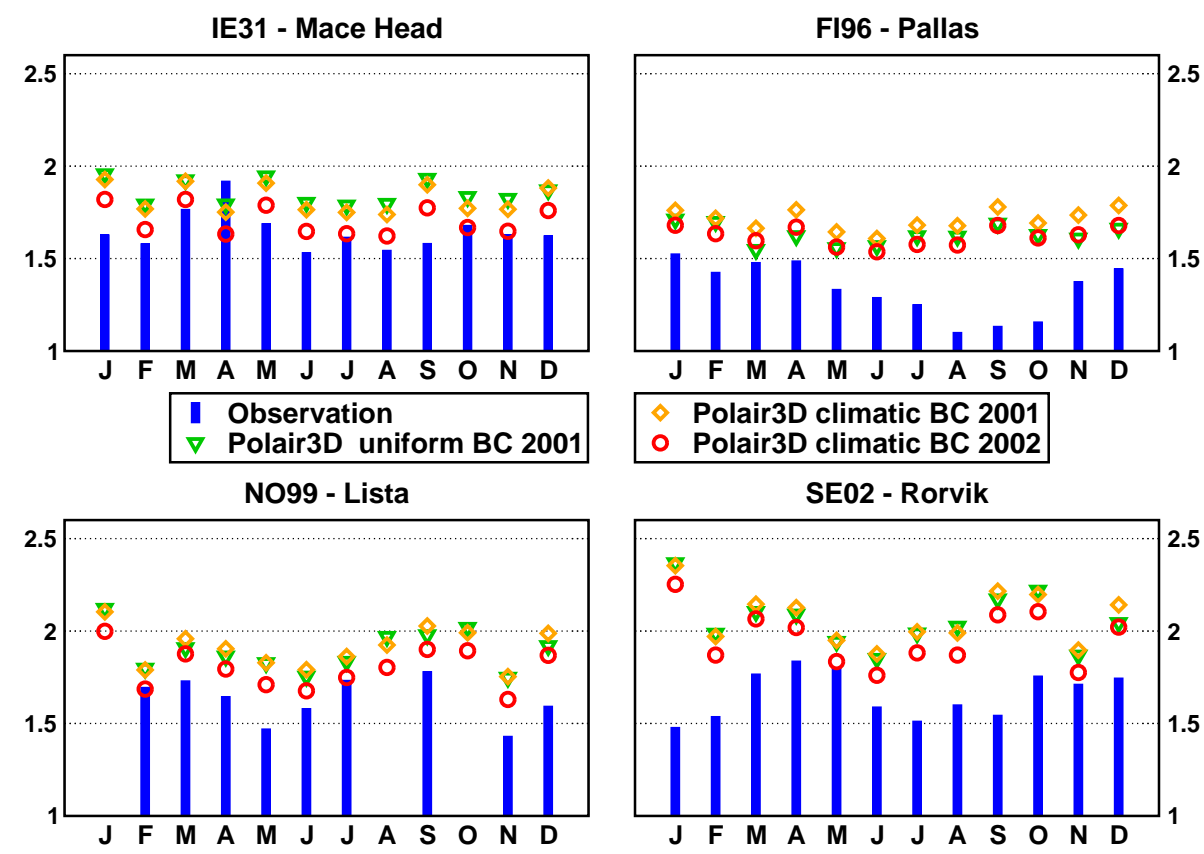

Fig. 4. Direct simulations results, in $\mathrm{ng} \mathrm{m}^{-3}$, for different boundary conditions (year 2001). The EMEP 2002 boundary conditions should clearly be preferred even for a 2001 simulation as boundary climatology.
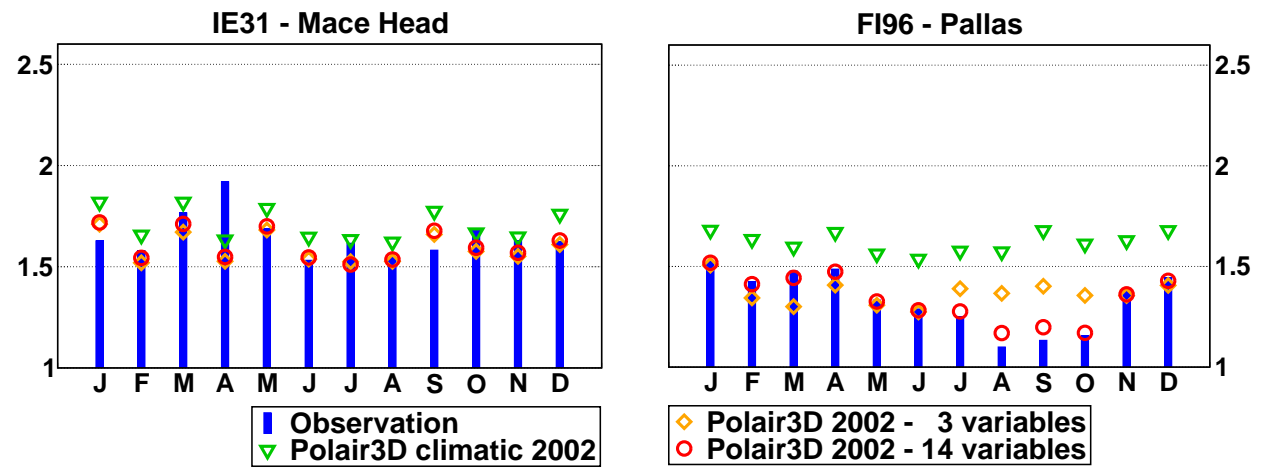

Fig. 5. Those two graphs display the assimilated concentrations, in $\mathrm{ng} \mathrm{m}^{-3}$, at Mace Head and Pallas. The first inversion with three parameters does not take into account intra-annual variability. The second inversion with fourteen parameters take into account the variability of the northern boundary conditions. The observed concentrations and the simulated ones are also given for comparison.

$[\mathbf{B}]_{\mathrm{ff}}=0.01 \mathcal{S}\left(\sum_{k \in \bar{\partial} \Omega_{+} \cap \bar{\partial} \Omega_{\mathrm{f}}} s_{k}^{\mathrm{f}} \boldsymbol{b}_{k}^{\mathrm{f} 2}\right)^{-1}$,

where $\mathrm{f}$ stands for $\mathrm{W}, \mathrm{N}$ or $\mathrm{E}, s_{k}^{\mathrm{f}}$ and $\boldsymbol{b}_{k}^{\mathrm{f}}$ are the surface and the background information (climatology) for the cell $k$ of the domain boundary $\mathrm{f}$ and $\mathcal{S}$ the total surface, $\mathcal{S}=\sum_{k \in \bar{\partial}_{+} \cap\left(\cup_{\mathrm{f}} \bar{\partial} \bar{\Omega}_{\mathrm{f}}\right)} s_{k}^{\mathrm{f}} \cdot \gamma$ is the trade-off (between the two departures) parameter and is dimensionless. Then, one obtains the normal equations giving the assimilated parameters $\lambda^{*}$

$\lambda^{*}=\lambda_{b}+\left[\gamma \mathbf{B}^{-1}+\mathbf{H}^{T} \mathbf{R}^{-1} \mathbf{H}\right]^{-1} \times \mathbf{H}^{T} \mathbf{R}^{-1}\left(\boldsymbol{\mu}-\boldsymbol{h}-\mathbf{H} \lambda_{b}\right)$.
After assimilation, the predicted values for the $\mu_{i}$ are given by $\boldsymbol{\mu}^{*}=\mathbf{H} \lambda^{*}+\boldsymbol{h}$. The results are shown on Fig. 5 (diamonds). Because these graphs show the predicted values on the sites which provided with the assimilated measurements, it is not surprising that the improvement is great compared to the direct simulation. Nonetheless only three boundary variables were assimilated to obtain these results.

More interestingly are the predicted elemental mercury concentrations on the stations Lista and Rörvik, whose measurements were not used in the inversion. The results are shown on Fig. 6 (diamonds).

The modelled concentrations are much closer to the observations than the simulation results without assimilation. 

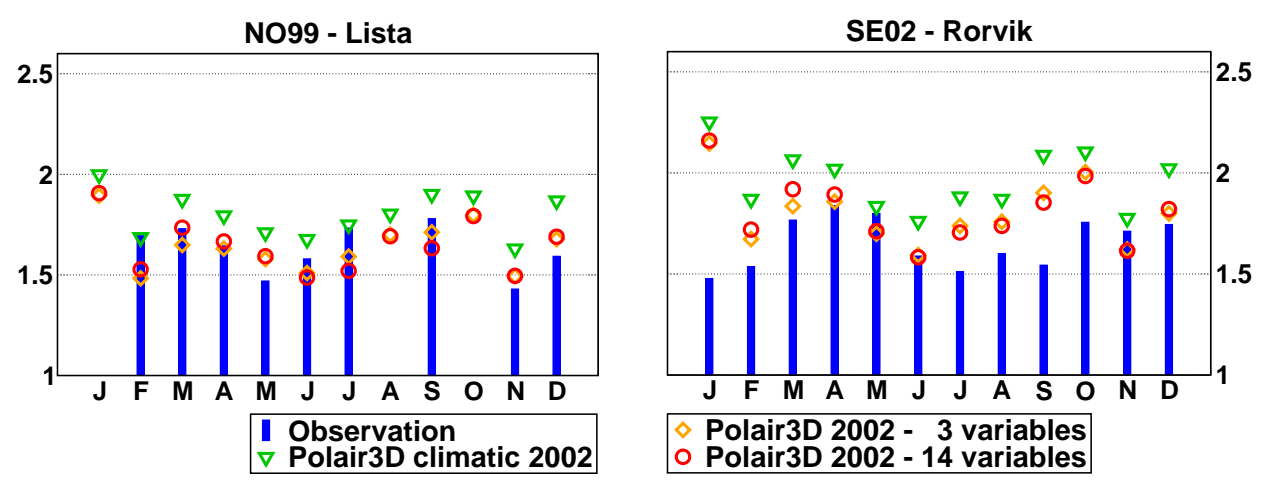

Fig. 6. Those two graphs display the assimilated concentrations, in $n g \mathrm{~m}^{-3}$, at Lista and Rörvik. The first inversion with three parameters does not take into account intra-annual variability. The second inversion with fourteen parameters take into account the variability of the northern boundary conditions. The observed concentrations and the simulated ones are also given for comparison.

Table 3. Assimilated coefficients $\lambda^{*}$ for boundary conditions, simple model.

\begin{tabular}{ccrrrr}
\hline \# Par. & West & \multicolumn{3}{c}{ North } & East \\
\hline 3 & 0.94 & & 0.81 & 0.78 \\
& & Jan -0.77 & Feb -0.82 & March -0.92 & \\
14 & 0.95 & April -0.85 & May -0.80 & June -0.73 & 0.84 \\
& & July -0.56 & Aug -0.33 & Sep -0.24 & \\
& & Oct -0.52 & Nov -0.78 & Dec -0.78 & \\
\hline
\end{tabular}

Hence the assimilation of observations on the first two sites has yielded benefits on the last two. The assimilated parameters are reported in Table 3.

It is however difficult to decide whether this improvement should be ascribed to the correction of a global bias only, or not. Fractional bias and fractional gross error (see Appendix) have been used as statistical indicators to evaluate the assimilation improvement with respect to the observed concentrations. The results are reported in Table 4. In particular, they concur with the improvements observed at Lista and Rörvik.

\subsection{Improving the monthly averaged boundary conditions}

It is noteworthy that several measurements on Pallas are not shadowed properly by the assimilated values. This is particularly striking for the summer season. This may stem from the mercury arctic depletion events (MDE). The modelling of this phenomenon is currently addressed in several works (see Ariya et al., 2004; Calvert and Lindberg, 2003). How to pragmatically represent the phenomenon within a hemispherical mercury model can be found in Christensen et al. (2004) or Travnikov and Ryaboshapko (2002). However, the arealimited domain used here does not encompass the Arctic. A way out of this problem is to invert monthly averaged bound-
Table 4. Fractional Bias (FB) and Fractional Gross Error (FE) between observed concentrations and modelled ones using various boundary conditions (in \%).

\begin{tabular}{|c|c|c|c|c|c|}
\hline & \multicolumn{2}{|c|}{$\begin{array}{l}\text { Mace Head } \\
\text { (IE31) }\end{array}$} & \multirow{2}{*}{$\begin{array}{l}\text { Pallas } \\
\text { (FI96) } \\
\text { FB FE }\end{array}$} & \multirow{2}{*}{$\begin{array}{c}\text { Lista } \\
\text { (NO99) } \\
\text { FB FE }\end{array}$} & \multirow{2}{*}{$\begin{array}{c}\text { Rörvik } \\
\text { (SE02) } \\
\text { FB FE }\end{array}$} \\
\hline & FB & FE & & & \\
\hline unif & -12 & 13 & -2020 & -1313 & $\begin{array}{ll}-21 & 21\end{array}$ \\
\hline climatic 2001 & -10 & 11 & -2525 & -1414 & $\begin{array}{ll}-22 & 22\end{array}$ \\
\hline 3 variables -2001 & 3 & 5 & -0.69 & 26 & $\begin{array}{ll}-8 & 10\end{array}$ \\
\hline 14 variables -2001 & 2 & 5 & -0.32 & 26 & -910 \\
\hline climatic 2002 & -3 & 6 & -1920 & $-8 \quad 8$ & $-17 \quad 17$ \\
\hline 3 variables -2002 & 4 & 5 & $\begin{array}{ll}-3 & 8\end{array}$ & 26 & $\begin{array}{ll}-8 & 10\end{array}$ \\
\hline 14 variables -2002 & 3 & 5 & $-0.4 \quad 2$ & 26 & -910 \\
\hline
\end{tabular}

ary conditions for the site Pallas which seems very sensitive to the phenomena. This will introduce intra-annual variability.

Taking into account monthly averaged concentrations implies using several adjoint solutions, each of them with a sampling function $\pi_{i}$ describing an emitter lasting one month. Again climatologies for the year 2002 will be used. Because the spin-up is of about two weeks, the inversion of parameters representing the last months of 2001 will not be affected by the initial condition. However, January or February parameters might. That is why the inversion is implemented as a two-year experiment. The adjoint solution are therefore calculated over two years. This lessens the impact of the initial condition. For the first year, the meteorological fields of 2001 are also used. The number of parameters to invert is 14. Two are related to the West and East boundary conditions: $\lambda_{\mathrm{W}}$ and $\lambda_{\mathrm{E}}$. Twelve others are used to parameterise month after month the North boundary condition $\lambda_{\mathrm{N}}=\left\{\lambda_{\mathrm{N}}^{i}\right\}_{i=1, \cdots, 12}$. If one assumes those twelve values 


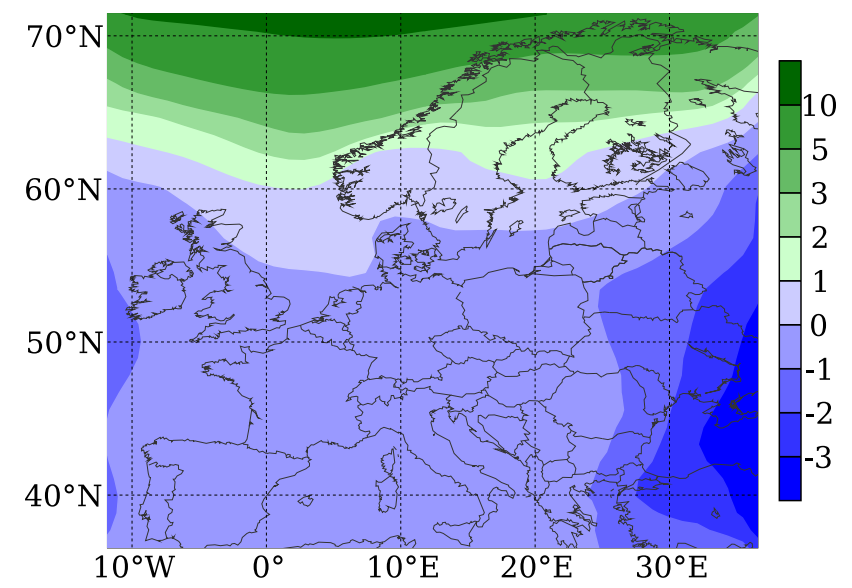

Fig. 7. Fractional bias (in \%) between annual mean concentrations of GEM resulting from two different simulations. The first one was performed using inverted boundary conditions for 2002 after assimilation with 3 variables (Sect. 4.2). The second simulation was performed using inverted boundary conditions for 2002 after assimilation with 14 variables (Sect. 4.3).

are uncorrelated, the inverse problem would then almost certainly be very ill-conditioned as a not too important change in a month boundary conditions can surely be compensated by other changes in the eleven other parameters. It is therefore necessary to correlate them with a correlation length that we have chosen to be three months:

$[\mathbf{B}]_{i j}=\mathrm{E}\left[\left(\lambda_{i}^{\mathrm{N}}-\lambda_{b}^{\mathrm{N}}\right)\left(\lambda_{j}^{\mathrm{N}}-\lambda_{b}^{\mathrm{N}}\right)\right]=e^{-\frac{|i-j|}{L}}$,

with $L \simeq 3$.

The results of the inversion is reported in Fig. 5 for the sites which provided with the measurements used in the assimilation (circles). The results for the two other sites are reported in Fig. 6. The improvement is spectacular only on the Pallas station. It is barely improved elsewhere. In particular, the discrepancy observed in summertime at Rörvik are not accounted for. It is likely that only the Pallas station is significantly sensitive to the mercury depletion event (it has the greatest latitude). If we look at the whole domain the use of the monthly means has strong influence only on its northern part. The Fig. 7 shows the fractional bias between annual mean modelised concentrations computed with 3 and 14 inverted variables. The values of the 14 parameters of the inversion are given in Table 3 .

On Table 5 are reported the yearly averaged concentrations at the four Nordic stations, observed, simulated, and simulated using assimilation techniques on the Mace Head and Pallas sites.

\subsection{Simple validation for $\gamma$}

So far, the measurements at Mace Head and Pallas were used to invert the boundary conditions parameters. However, an

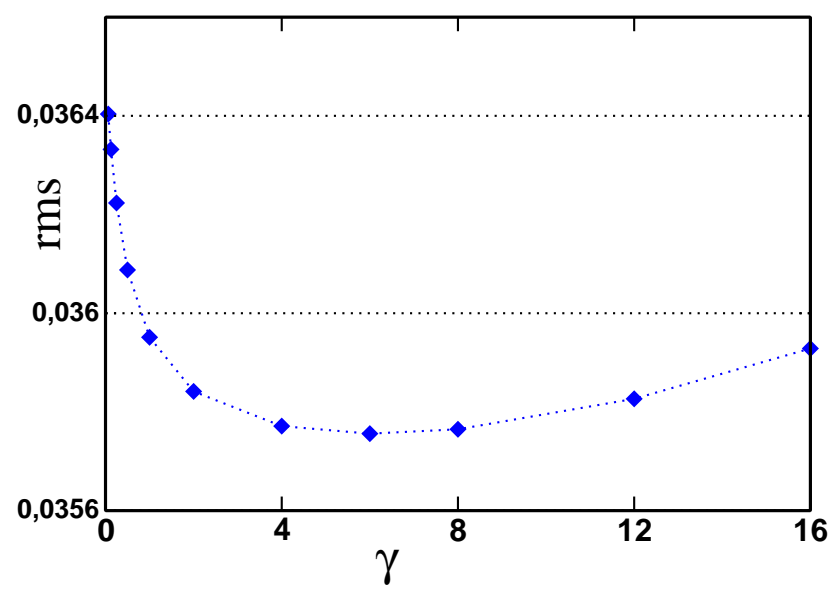

Fig. 8. Cross-validation of parameter $\gamma$. The root mean square (in $\mathrm{n} \mathrm{m}^{-3}$ ) of the departure of the forecast values to the observed values at Lista and Rörvik is plotted as a function of $\gamma$.

ad-hoc parameter $\gamma$ was used to control the relative contributions of variances of observations and background to the inversion.

The inversion can be repeated for several values of $\gamma$. The performance (r.m.s.) of the predicted concentrations using these inversions can then be assessed at Lista and Rörvik, the other two EMEP gaseous elemental mercury monitoring stations. If $\gamma$ is very large, then the solution is forced by the background, the data do not tell more than the prior information and a strong mismatch is expected between the observed and predicted concentrations at Lista and Rörvik. On the other hand, if $\gamma$ is small, the inversion only aims at giving an account of the observed concentrations at Mace Head and Pallas, even accounting for unrealistic errors. Those errors propagate by forecast to the other two stations. The forecast on Lista and Rörvik is therefore expected to be affected in this limit. As a consequence, there may be an optimal value of $\gamma$ in between those two limits.

The result of this test is represented on Fig. 8. There is an optimal value of about $\gamma \simeq 6$. All previous inversion were performed with $\gamma=4$ and we conclude that this guess was a good one, since the differences between forecasts with $\gamma=4$ and $\gamma=6$ are small.

\subsection{Improving emissions inventory}

It has been demonstrated that it is possible to improve significantly predicted values of mercury dispersion in an area limited model by using inverse modelling on the boundary conditions. It should be possible to use a similar approach working on the emissions (natural or anthropogenic). The tests we have performed are negative in this respect. The related inverse problem is much too ill-conditioned (testified by the weak singular values of $\mathbf{H}$ ), when using data from the four Nordic EMEP stations. This can be understood by the 
Table 5. Annual average concentration (in $\mathrm{ng} \mathrm{m}^{-3}$ ).

\begin{tabular}{|c|c|c|c|c|}
\hline observation & $\begin{array}{c}\text { Mace Head } \\
\text { (IE31) } \\
1.64\end{array}$ & $\begin{array}{c}\text { Pallas } \\
\text { (FI96) } \\
1.32\end{array}$ & $\begin{array}{c}\text { Lista } \\
\text { (NO99) } \\
1.65\end{array}$ & $\begin{array}{c}\text { Rörvik } \\
\text { (SE02) } \\
1.66\end{array}$ \\
\hline uniform 2001 & 1.86 & 1.63 & 1.90 & 2.05 \\
\hline climatic 2001 & 1.82 & 1.71 & 1.91 & 2.07 \\
\hline 3 variables -2001 & 1.60 & 1.34 & 1.65 & 1.80 \\
\hline 14 variables -2001 & 1.61 & 1.34 & 1.65 & 1.81 \\
\hline climatic 2002 & 1.71 & 1.62 & 1.80 & 1.96 \\
\hline 3 variables -2002 & 1.59 & 1.37 & 1.64 & 1.80 \\
\hline 14 variables -2002 & 1.61 & 1.34 & 1.65 & 1.81 \\
\hline
\end{tabular}

too weak sensitivities of those stations to the European emissions. At the stations, the actual contributions of the emissions represent $8 \%, 5 \%, 12 \%$ and $16 \%$ of the total gaseous mercury measured (to compare with, for instance, the Topolniky site (SK07) with a ratio of $40 \%$ ).

To improve emissions inventory using inverse modelling, one would therefore need stations where the emissions influence is significant (central European locations). Unfortunately, to our knowledge, no measurement of gaseous mercury is available on a regular basis, except for the measurements performed at the stations already introduced, but where the emissions influence is too weak. We believe having such data on mercury would greatly help modellers.

Moreover it is not so much GEM modelling itself which is at stake, but the improvement of models which ultimately predict deposition of oxidised forms of mercury.

\section{Extension to a complex chemical model}

So far, the inverse modelling approach presented here was based on a mercury dispersion model relying on the Petersen scheme. So that oxidised species were not properly modelled. In a first approximation, this was however acceptable since the boundary conditions to be inverted were concerned with the barely reactive gaseous elemental mercury.

Nevertheless it is possible to extend this inverse modelling approach to cope with a more complex mercury chemistry. It is expected that this would be more relevant to measurement stations in the vicinity of anthropogenic sources. Out of the four EMEP stations considered here, this could be relevant to Rörvik as it is sensitive to northern European pollution. It was shown in Roustan and Bocquet (2006) that the adjoint analysis (needed for the inverse approach) can be extended to cope with oxidised species and their chemistry. Here, we rely on a seven aggregate species model developed in Roustan et al. (2005) and Roustan (2005) in both gaseous and aqueous phases. Those considered in the gaseous phase are $\mathrm{Hg}(0)$, $\mathrm{HgO}, \mathrm{Hg}(\mathrm{OH})_{2}$ and $\mathrm{HgCl}_{2}$ and their sum will be noted as total gaseous mercury (TGM) in the following.
From the modellers perspective, this chemistry is linear in the mercury species, although it involves other species such as $\mathrm{SO}_{2}, \mathrm{O}_{3}, \mathrm{OH}$, etc, which are forced into the model. The chemistry and transport equation are extended to:

$$
\frac{\partial \boldsymbol{c}}{\partial t}+\operatorname{div}(\boldsymbol{u c})-\operatorname{div}(\mathbf{K} \nabla \boldsymbol{c})+\mathbf{\Lambda} \boldsymbol{c}+\mathbf{M} \boldsymbol{c}=\sigma .
$$

$\boldsymbol{c}$ is the vector of mercury species (seven components in the model mentioned above). $\boldsymbol{\Lambda}$ is the diagonal matrix of the scavenging coefficient (species-dependent). $\mathbf{M}$ is the kinetic matrix describing the first-order (in mercury) chemistry and depends on forced fields of other species concentration.

To generalise the adjoint analysis performed with the Petersen model, it is convenient to introduce the canonical scalar product in the space of mercury species: $\langle\boldsymbol{x}, \boldsymbol{y}\rangle=\boldsymbol{x}^{T} \boldsymbol{y}$. The measurement equation is now:

$\mu_{i}=\int_{\Omega} \mathrm{d} t \mathrm{~d} \boldsymbol{x}\left\langle\boldsymbol{\pi}_{i}(\boldsymbol{x}, t), \boldsymbol{c}(\boldsymbol{x}, t)\right\rangle$.

The sampling function $\boldsymbol{\pi}_{i}$ is a vector in the space of species, and describes how each of the species is sampled. Even if the focus is on GEM in this work, $\boldsymbol{\pi}_{i}$ will have four non-zero components since genuine measurements concern TGM. If we were able to distinguish the GEM component of the measurement we could work with a sampling function having only one non-zero component. The retro-transport equation generalises to:

$-\frac{\partial \boldsymbol{c}_{i}^{*}}{\partial t}-\operatorname{div}\left(\boldsymbol{u} \boldsymbol{c}_{i}^{*}\right)-\operatorname{div}\left(\mathbf{K} \nabla \boldsymbol{c}_{i}^{*}\right)+\boldsymbol{\Lambda} \boldsymbol{c}_{i}^{*}+\mathbf{M}^{T} \boldsymbol{c}_{i}^{*}=\boldsymbol{\pi}_{i}$

For a concentration measurement such as the one described by Eq. (17), the adjoint analysis is similar and one obtains

$$
\begin{aligned}
\mu_{i}= & \int_{\Omega} \mathrm{d} t \mathrm{~d} \boldsymbol{x}\left\langle\boldsymbol{c}_{i}^{*}, \boldsymbol{\sigma}\right\rangle+\int_{\partial \Omega_{0}} \mathrm{~d} \boldsymbol{x}\left\langle\boldsymbol{c}_{i}^{*}, \boldsymbol{c}\right\rangle \\
& +\int_{\partial \Omega_{b}} \mathrm{~d} t \mathrm{~d} \boldsymbol{S} \cdot\left(\left\langle\boldsymbol{c}, \boldsymbol{J}_{i}^{*}\right\rangle-\left\langle\boldsymbol{c}_{i}^{*}, \boldsymbol{J}\right\rangle\right) \\
& -\int_{\partial \Omega_{+}} \mathrm{d} t \mathrm{~d} \boldsymbol{S} \cdot\left(\left\langle\boldsymbol{c}_{i}^{*}, \boldsymbol{c}\right\rangle \boldsymbol{u}\right) .
\end{aligned}
$$

It has been checked that the non-linearities introduced by the improved chemical scheme (some threshold being used to treat the aqueous phase) result in a very weak violation of the additivity principle. As in the case of the Petersen scheme (Roustan and Bocquet, 2006), the difference between a single multiple-component run and the sum of single-component runs, for each gaseous species, does not exceed $0.1 \%$.

Moreover, the error committed between the direct and the indirect calculations of the contributions to the mercury gaseous modelled concentration at Mace Head (IE31) and Pallas (FI96) has been estimated. The results are presented in Table 6. The approximations made in the computation of the adjoint solution and when taking the numerical model to be linear seem fairly contained. 

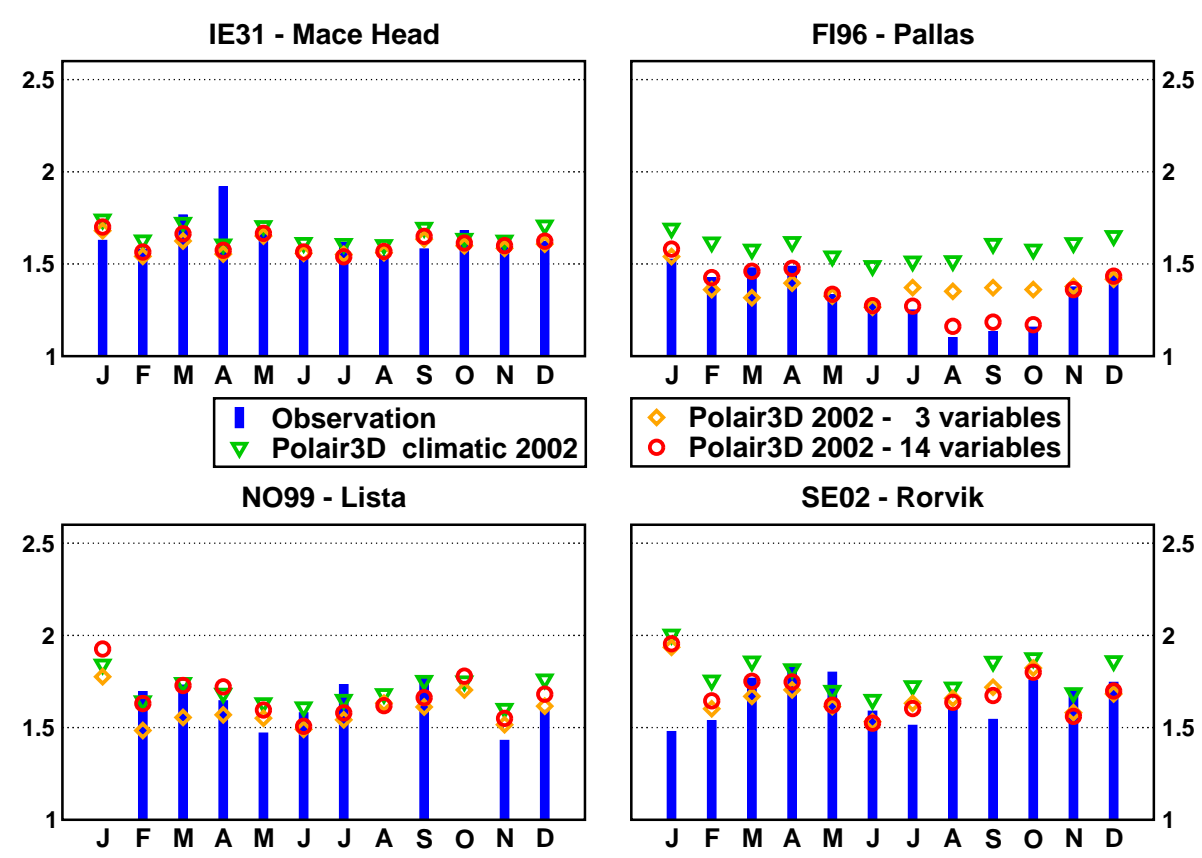

Fig. 9. Those four graphs display the assimilated concentrations of total gaseous mercury, in $\mathrm{ng} \mathrm{m}^{-3}$, at Mace Head, Pallas, Lista and Rörvik obtained with a more realistic model. The first inversion with three parameters does not take into account intra-annual variability. The second inversion with fourteen parameters take into account the variability of the northern boundary conditions. The observed concentrations and the simulated ones are also given for comparison.

\subsection{Improving GEM boundary conditions using a complex} chemical scheme

We assume that at the boundary, far from anthropogenic sources, the fraction of oxidised species is low. It is set to zero. This point will be discussed more thoroughly in the next section. The parameters $\lambda$ are therefore only scaling the concentration of GEM at the boundaries. One is therefore interested in the sensitivity of the TGM measurement to the gaseous incoming elemental mercury through one of the borders:

$\frac{\delta \mu_{i}}{\delta \lambda_{\mathrm{f}}}=\sum_{k \in \bar{\partial} \Omega_{+} \cap \bar{\partial} \Omega_{\mathrm{f}}}\left[c_{i,(\mathrm{TGM}, \mathrm{GEM})}^{*}\right]_{k} c_{k}^{\mathrm{f}} u_{k}$,

where $\left[c_{i,(\mathrm{TGM}, \mathrm{GEM})}^{*}\right]$ is the GEM component of $c_{i}^{*}$ computed with a TGM sampling function and $\mathrm{f}$ stands for $\mathrm{W}, \mathrm{E}$ or $\mathrm{N}$. This defines the matrix $\mathbf{H}$ according to Sect. 4.2. And the same data assimilation procedure can be applied. The measurement equation $\boldsymbol{\mu}=\mathbf{H} \boldsymbol{\lambda}+\boldsymbol{h}$ requires also a different definition for $\boldsymbol{h}$, which takes into account prior emissions of oxidised species. Aside from these differences, the cost function remains formally the same. $\gamma$ is chosen to be $\gamma \simeq 4$ again. It has been checked that $\gamma$ is not far from an optimal value as it was the case in the simple chemistry inversion problem. The results are reported in Fig. 9. The inverted parameters are given in Table 7.

The statistics of those results are reported in Table 8. There are clear improvements due to the improved chemical model.
Table 6. Contribution to gaseous mercury concentrations in $\mathrm{ng} \mathrm{m}^{-3}$ for year 2001 over Europe, as computed from direct simulations (right), and from adjoint simulations (left).

\begin{tabular}{cccc}
\hline Station & Winds & Emissions & Total \\
\hline $\begin{array}{c}\text { Mace Head } \\
\text { (IE31) }\end{array}$ & $1.715-1.709$ & $0.119-0.098$ & $1.834-1.807$ \\
Pallas (FI96) & $1.527-1.524$ & $0.065-0.066$ & $1.592-1.588$ \\
\hline
\end{tabular}

This was expected for the Rörvick station. It has been emphasised that the northern European emissions have some influence on this station, so that the chemical reactions play a significant role in the mercury dispersion. The assimilated results are better for Pallas (FI96) and Rörvik (SE02), but they are slightly degraded for Mace Head (IE31) and Lista (NO99). Nevertheless this result is not really surprising. The modelled concentrations estimated with the climatic boundary conditions are only slightly overestimated at Mace Head and largely at Pallas. The assimilation process leads to decrease boundary conditions both in North and West (see Table 7). The contributions to the modelled concentrations are of the same order at Pallas, $0.44 \mathrm{ng} \mathrm{m}^{-3}$ for the western boundary and $0.57 \mathrm{ng} \mathrm{m}^{-3}$ for the northern one. 
Table 7. Assimilated coefficients $\lambda^{*}$ for boundary conditions, complex model.

\begin{tabular}{ccrcrc}
\hline \# Par. & West & \multicolumn{3}{c}{ North } & East \\
\hline 3 & 0.97 & & 0.85 & 0.83 \\
& & Jan -0.90 & Feb -0.91 & March -0.93 & \\
14 & 0.98 & April -0.90 & May -0.82 & June -0.78 & 0.88 \\
& & July -0.65 & Aug -0.47 & Sep -0.41 & \\
& & Oct -0.57 & Nov -0.81 & Dec -0.70 & \\
& & & & &
\end{tabular}

\subsection{Possible improvement of the other inputs}

We have assumed until now that boundary conditions of oxidised species were negligible. Accordind to the measurements presented in Ebinghaus et al. (1999) and Aspmo et al. (2005) this assumption is not fully realistic. RGM species would represent barely more than 2 or $3 \%$ of the averaged TGM concentrations in background conditions. Yet this is not negligible in comparison to the difference between observed and modelised concentrations (see Table 8). The error commited should be stronger for the stations near the border of the domain (IE31, FI96). However depending on the life time of RGM species it could be substantial in the mainland also. Nevertheless, according to the results presented in Roustan and Bocquet (2006), this should not be the case with the model used here. Obviously, the error commited on IE31 and FI96 are partially "transfered" to NO99 and SE02 through the assimilation process.

Unfortunately, direct assimilation of measured concentrations of TGM in order to improve the boundary conditions of oxidised species cannot be achieved with the available data. TGM concentrations are too poorly sensitive to the model parameters to invert (the inverse problem on the oxidised species only would be too ill-conditioned). At this point measurements of oxidised species could be useful, all the more since advances have been made in reactive gaseous and particulate mercury sampling (Landis et al., 2002).

Another way to improve boundary conditions of oxidised species would consist in using deposition measurement data. Since deposition fluxes are much more sensitive to RGM concentrations (Roustan and Bocquet, 2006) the inverse problem could be better conditioned. In this case, the precedent assumption concerning the boundary conditions of oxidised species is much less satisfactory and should be avoided. This is however beyond the scope of this work.

Nevertheless, it has been shown that the emission speciation was the key parameter for determining the mercury deposition fluxes in source areas (Pai et al., 1999). A similar problem is met: more sampling stations with a central European location are needed. We believe that regular measurements of TGM and RGM could be used to improve efficiently the mercury emission inventory.
Table 8. Fractional Bias (FB) and Fractional Gross Error (FE) between observed concentrations and modelled ones using various boundary conditions with the complex chemistry model (in \%).

\begin{tabular}{|c|c|c|c|c|c|c|}
\hline & \multicolumn{2}{|c|}{$\begin{array}{l}\text { Mace Head } \\
\quad(\text { IE31) }\end{array}$} & \multirow{2}{*}{$\begin{array}{l}\text { Pallas } \\
\text { (FI96) } \\
\text { FB FE }\end{array}$} & \multicolumn{2}{|c|}{$\begin{array}{c}\text { Lista } \\
\text { (NO99) }\end{array}$} & \multirow{2}{*}{$\begin{array}{l}\text { Rörvik } \\
\text { (SE02) } \\
\text { FB FE }\end{array}$} \\
\hline & FB & $\mathrm{FE}$ & & FB & $\mathrm{FE}$ & \\
\hline climatic 2002 & -0.6 & 5 & $-17 \quad 18$ & -3 & 5 & -8 \\
\hline 3 variables -2002 & 3 & 5 & -58 & 4 & 7 & -2 \\
\hline 14 variables -2002 & 3 & 4 & $\begin{array}{ll}-3 & 4\end{array}$ & 5 & 7 & -2 \\
\hline
\end{tabular}

As previously mentioned, the deposition fields are of a greater interest than air concentration. Particulate mercury represents an appreciable part of the total mercury mass deposited. Measurements for this "species" would therefore be very useful. More generally, observations specific to genuine oxidised mercury species $\left(\mathrm{HgO}, \mathrm{Hg}(\mathrm{OH})_{2}, \mathrm{HgCl}_{2}\right.$, etc) or vertical distributions in the atmosphere could be helpful.

\section{Conclusions}

In this paper, we have attempted to correct some of the flaws inherent to regional modelling of mercury dispersion. Although using a regional model allows for a fine resolution description, it is very sensitive to external forcing fields, mainly boundary conditions (incoming mercury). To compensate for this weakness, we have assimilated observations of gaseous mercury to improve these boundary conditions, using the regional model POLAIR3D. It was shown to improve forecasts for gaseous mercury over Europe, not only on the monitoring stations which provided the assimilated data, but also on the others. We have resorted to the linearity of the dispersion and to the adjoint techniques to establish the linear relation between the concentrations at the monitoring stations and the forcing fields.

The first tests were performed for annual boundary conditions. The method presented here is applied to averaged fields. Yet, external influences, such as mercury depletion event, were accounted for by using monthly boundary conditions. We hope that a finer temporal descriptions of the measurements (which are available) could be used to improve the description of the boundary conditions. However this remains to be checked and such an approach requires more consequent computational resources (since one adjoint simulation is needed for each spatially and temporally located measurement). Moreover, one of our motivation is impact studies for which we believe that annual and monthly time scale are relevant.

The improvement on the GEM concentrations forecast with the Petersen scheme model and using assimilated boundary conditions is significant. It was bound to be so for the two EMEP stations which provided with the assimilated 
data but this conclusion still holds for the two remaining stations. There is however no significant improvement on the two last stations (whose measurements were not assimilated), when using the complex scheme model, as compared to the complex model without assimilated boundary conditions. This might be ascribed to the absence of well known boundary conditions for the oxidised species.

We have concluded that the mercury observation network is insufficient to take full benefit of the approach. In particular it does not allow to invert emissions with confidence. This lack of data may be compensated in a near future since this is part of the EMEP monitoring strategy for the next four years.

It was shown that the adjoint analysis could be performed on oxidised mercury, when using a realistic chemistry. Hence, there exists a linear relation between the modelled oxidised mercury concentrations and the forcing fields. In this work were performed first experiments of inversion using a realistic model, but only inverting gaseous elemental mercury. The advantage of it on the simple model approach turned out not to be obvious. In Roustan and Bocquet (2006), we have shown that the sensitivity analysis could be extended to measurements of deposited mercury, not only air content measurements. Inversions of measurements of deposited mercury are therefore, in principle, possible. It should be investigated in future works, since many more measurements involving deposited oxidised mercury are available from the EMEP monitoring stations.

\section{Appendix A}

\section{Statistical indicators}

Here are defined the statistical indicators used in this work. Consider a set of concentration measurements $\mu_{i=1, \cdots, p}$, and a set of predicted values for those measurements $c_{i=1, \cdots, p}$. Define the means

$\bar{\mu}=\frac{1}{p} \sum_{i=1}^{p} \mu_{i} \quad$ and $\quad \bar{c}=\frac{1}{p} \sum_{i=1}^{p} c_{i}$.

The bias and the fractional bias (FB) are defined by

$\bar{\mu}-\bar{c}$ and $2 \frac{\bar{\mu}-\bar{c}}{\bar{\mu}+\bar{c}}$,

and the fractional gross error $(\mathrm{FE})$ is

$\frac{2}{p} \sum_{i=1}^{p}\left|\frac{c_{i}-\mu_{i}}{c_{i}+\mu_{i}}\right|$

The normalised root mean square is

$$
\sqrt{\frac{1}{p} \sum_{i=1}^{p} \frac{\left(\mu_{i}-c_{i}\right)^{2}}{\bar{\mu} \bar{c}}}
$$

and eventually the individually normalised root mean square is

$$
\sqrt{\frac{1}{p} \sum_{i=1}^{p} \frac{\left(\mu_{i}-c_{i}\right)^{2}}{\mu_{i} c_{i}}} .
$$

Acknowledgements. The authors wish to thank I. Ilyin and the MSC-E team for kindly providing results of the MSCE model. Y. Roustan acknowledges financial support from ADEME and EDF.

Edited by: Y. Balkanski

\section{References}

Ariya, P. A., Dastoor, A. P., Amyot, M., Schroeder, W. H., Barrie, L., Anlauf, K., Raofie, F., Ryzhkov, A., Davignon, D., Lalonde, J., and Steffen, A.: The Arctic: a sink for mercury, Tellus, 56B, 397-403, 2004.

Aspmo, K., Gauchard, P. A., Steffen, A., Temme, C., Berg, T., Bahlmann, E., Banic, C., Dommergue, A., Ebinghaus, R., Ferrari, C., Pirrone, N., Sprovieri, F., and Wibetoe, G.: Measurements of atmospheric mercury species during an international study of mercury depletion events at Ny-Ålesund, Svalbard, spring 2003. How reproducible are our present methods?, Atmos. Environ., 39, 7607-7619, 2005.

Baer, M. and Nester, K.: Parameterization of trace gas dry deposition velocities for a regional mesoscale diffusion model, Ann. Geophys., 10, 912-923, 1992, http://www.ann-geophys.net/10/912/1992/.

Boutahar, J., Lacour, S., Mallet, V., Quélo, D., Roustan, Y., and Sportisse, B.: Development and validation of a fully modular platform for numerical modelling of air pollution: POLAIR, Int. J. Environ. Pollut., 22(1/2), 17-28, 2004.

Brook, J. R., Zhang, L., Di-Giovanni, F., and Padro, J.: Description and evaluation of a model of deposition velocities for routine estimates of air pollutant dry deposition over North America. Part I: model development, Atmos. Environ., 33, 5037-5051, 1999.

Bullock, O. R. and Brehme, K. A.: Atmospheric mercury simulation using the CMAQ model: formulation description and analysis of wet deposition results, Atmos. Environ., 36, 2135-2146, 2002.

Calvert, J. G. and Lindberg, S. E.: A modeling study of the mechanism of the halogen-ozone-mercury homogeneous reactions in the troposphere during the polar spring, Atmos. Environ., 37, 4467-4481, 2003.

Christensen, J. H., Brandt, J., Frohn, L. M., and Skov, H.: Modelling of mercury with the Danish Eulerian Hemispheric Model, Atmos. Chem. Phys., 4, 2251-2257, 2004, http://www.atmos-chem-phys.net/4/2251/2004/.

Ebinghaus, R., Jennings, S. G., Schroeder, W. H., Berg, T., Donaghy, T., Guentzel, J., Kenny, C. Kock, H. H., Kvietkus, K., Landing, W., Mhleck, T. Munthe, J., Prestbo, E. M., Schneeberger, D., Slemr, F., Sommar, J., Urba, A., Wallschläger, D., and Xiao, Z.: International field intercomparison measurements of atmospheric mercury species at Mace Head, Ireland, Atmos. Environ., 33, 3063-3073, 1999. 
Hicks, B. B. and Liss, P. S.: Transfer of $\mathrm{SO}_{2}$ and other reactive gases across the air-sea interface, Tellus, 28, 348-354, 1976.

Ilyin, I., Ryaboshapko A., and Travnikov, O.: Heavy metal contamination on European and Hemisperical scale, EMEP Status report 3/2002, 2002.

Ilyin, I., Travnikov, O., Aas, W., and Uggerud, H. T.: Heavy metals: transboundary pollution of the environment, EMEP Status report 2/2003, 2003.

Landis, M. S., Stevens, R. K., Schaedlich, F., and Prestbo, E. M.: Development and characterization of annular denuder methodology for the measurement of divalent inorganic reactive gaseous mercury in ambient air, Environ. Sci. Technol., 36, 3000-3009, 2002.

Lin, X. and Tao, Y.: A numerical modelling study on regional mercury budget for eastern North America, Atmos. Chem. Phys., 3, 535-548, 2003, http://www.atmos-chem-phys.net/3/535/2003/.

Lindqvist, O. and Rodhe, H.: Atmospheric mercury - a review, Tellus, 37B, 136-159, 1985.

Pai, P., Karamchandani, P., and Seigneur, C.: Sensitivity of simulated atmospheric mercury concentrations and depositions to model input parameters, J. Geophys. Res., 104, 13 855-13 868, 1999.

Petersen, G, Iverfeldt, A., and Munthe, J.: Atmospheric mercury species over central and northern Europe. Model calculations and comparison with observations from the nordic air and precipitation network for 1987 and 1988, Atmos. Environ., 29, 47-67, 1995.

Roustan, Y., Bocquet, M., Musson Genon, L., and Sportisse, B.: Modeling atmospheric mercury at European scale with the Chemistry Transport Model POLAIR3D, Proceedings of Gloream 2004, available at: http://www2.dmu.dk/atmosphericenvironment/gloream/ 2gloreameurasapproceedings.pdf, 2005.

Roustan, Y. and Bocquet, M.: Sensitivity analysis for mercury over Europe, J. Geophys. Res., 111, D14304, doi:10.1029/2005JD006616, 2006.

Roustan, Y.: Modélisation de la dispersion atmosphrique du mercure, du plomb et du cadmium à l'échelle européenne. $\mathrm{PhD}$ Thesis, École Nationale des Ponts et Chaussées, Paris, 2005.
Ryaboshapko, A., Ilyin, I., Gusev, A., and Afinogenova, O.: Mercury in the atmosphere of Europe: concentrations, deposition patterns, transboundary fluxes, EMEP/MSC-E Report 7/1998, 1998.

Ryaboshapko, A., Bullock, R., Ebinghaus, R., Ilyin, I., Lohman, K., Munthe, J., Petersen, G., Seigneur, C., and Wängberg, I.: Comparison of mercury chemistry models, Atmos. Environ., 36, 3881-3898, 2002.

Ryaboshapko, A., Artz, R., Bullock, R., Christensen, J., Cohen, M., Dastoor, A., Davignon, D., Draxler, R., Ebinghaus, R., Ilyin, I., Munthe, J., Petersen, G., and Syrakov, D.: Intercomparison study of numerical models for long-range atmospheric transport of mercury. Stage II. Comparison of modeling results with observations obtained during short-term measuring campaigns, MSC-E Technical Report 1/2003, 2003.

Sartelet, K., Boutahar, J., Quélo, D., Coll, I., Plion, P., and Sportisse, B: Development and Validation of a 3D ChemistryTransport Model, POLAIR3D, by Comparison with Data from ESQUIF Campaign, Proceedings of the 6th Gloream workshop: Global and regional atmospheric modeling, 140-146, 2002.

Seigneur, C., Karamchandani, P., Lohman, K., Vijayaraghavan, K., and Shia, R. L.: Multiscale modeling of the atmospheric fate and transport of mercury, J. Geophys. Res., 106, 27 795-27 809, 2001.

Seigneur, C., Karamchandani, P., Vijayaraghavan, K., Lohman, K., Shia, R., and Levin, L.: On the effect of spatial resolution on atmospheric mercury modeling, The Science of the Total Environment, 304, 73-81, 2003.

Spee, E.: Numerical methods in global transport models, PhD Thesis, Univ. Amsterdam, 1998.

Sportisse, B., Boutahar, J., Debry, E., Quélo, D., and Sartelet, K.: Some tracks in Air Pollution Modeling. POLAIR: a numerical platform for air pollution modeling, RACSAM Journal of the Spanish Science Academy, Real Academia de Ciencias de Espana, 96, 507-528, 2002.

Travnikov, O. and Ryaboshapko, A.: Modelling of mercury hemispheric transport and depositions, MSC-E Tech. Report 6/2002, 2002.

Wesely, M. L. and Hicks, B. B.: A review of the current status of knowledge on dry deposition, Atmos. Environ., 34, 2261-2281, 2000 . 